\title{
Traditional herbal prescription LASAP-C inhibits melanin synthesis in B16F10 melanoma cells and zebrafish
}

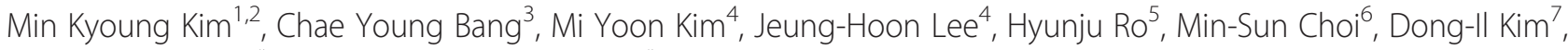
Young Pyo Jang ${ }^{1,2^{*}}$ (D) and Se Young Choung ${ }^{1,3^{*}}$

\begin{abstract}
Background: In this study, the anti-melanogenesis efficacy of clinically used herbal prescription LASAP-C, which consists of four herbal medicines-Rehmanniae Radix Crudus, Lycii Fructus, Scutellariae Radix, and Angelicae Dahuricae Radix, was investigated.

Methods: The chemical profile of LASAP-C was established by conducting ultra-performance liquid chromatographyelectrospray ionization-mass spectrometry. Anti-melanogenic efficacy was evaluated by tyrosinase, tyrosinase-related protein (TRP)-1, and TRP-2 expression in B16F10 melanoma cells. In vivo evaluation was performed by using zebrafish model.

Results: Molecular evidences suggested that melanin synthesis was inhibited via the down-regulation of tyrosinase, tyrosinase-related protein (TRP)-1, and TRP-2 expression in B16F10 melanoma cells treated with LASAP-C. The anti-melanogenesis efficacy was also confirmed in vivo by using the zebrafish model.

Conclusion: The results of this study provide strong evidences that LASAP-C can be used as an active component in cosmeceutical products for reducing excess pigmentation in the human skin.
\end{abstract}

Keywords: LASAP-C, Herbal prescription, Melanin, Tyrosinase, TRP-1, TRP-2, Zebrafish

\section{Background}

Melanin, the major pigment of human skin, is secreted by melanocytes in the basal layer of the epidermis. It is known to be overproduced in the cases of chronic sun exposure, melasma, or other hyperpigmentation diseases $[1,2]$. Melanin biosynthesis can be inhibited by avoiding ultraviolet (UV) exposure, preventing melanocyte metabolism and proliferation, and blocking activities of enzymes such as tyrosinase [3]. Among the various causes of excessive melanin synthesis, UV radiation has been known to induce the formation of reactive oxygen species (ROS) in the skin, such as singlet oxygen and superoxide anion, promoting biological damage in exposed tissues via ironcatalyzed oxidative reactions [4-6]. These ROS enhance

\footnotetext{
* Correspondence: ypjang@khu.ac.kr; sychoung@khu.ac.kr

${ }^{1}$ Department of Life and Nanopharmaceutical Sciences, College of Pharmacy, Kyung Hee University, Hoegi-dong, Dongdaemun-gu, Seoul 130-701, South Korea

Full list of author information is available at the end of the article
}

melanin biosynthesis and DNA damage and might induce melanocyte proliferation [7-9].

Tyrosinase, a copper-containing monooxygenase, is a key enzyme that catalyzes two major reactions-hydroxylation of tyrosine and oxidation of L-dopa-of melanin synthesis by melanocytes [10]. Dopa oxidation produces a highly reactive intermediate that is further oxidized to form melanin via a free radical-coupling pathway [10].

In this study, we evaluated the anti-melanogenesis effect of the traditional herbal prescription LASAP-C, which was found to be effective in an in vitro tyrosinase assay. LASAP-C was derived from Korean traditional herbal prescriptions and utilized as skin tone brightening agent in local Clinique [11]. LASAP-C is composed of four herbal medicines Rehmanniae Radix Crudus, Lycii Fructus, Scutellariae Radix, and Angelicae Dahuricae Radix. We used B16F10 melanoma cells as a model system to investigate the effects of LASAP-C on antimelanogenesis and evaluated the underlying molecular 
mechanisms such as the effect on the expression of tyrosinase, tyrosinase-related protein (TRP)-1, and TRP-2. We also determined the inhibitory effect of LASAP-C on melanin synthesis by using an in vivo model of zebrafish.

\section{Methods}

\section{Preparation of LASAP-C water extract}

The raw material used for preparing LASAP-C (235 g) was Rehmannia glutinosa Libosch. var. purpurea Makino (Scrophulariaceae; root, 100 g; voucher specimen number: DUMCKM2015-040), Lycium chinense Mill. (Solanaceae; fruit, $50 \mathrm{~g}$; voucher specimen number: DUMCKM2015008), Scutellaria baicalensis Georgi (Labiatae; root, 50 g; voucher specimen number: DUMCKM2015-081), and Angelica dahurica Bentham et Hooker f. (Umbelliferae; root, 35 g; voucher specimen number: DUMCKM2015-031). The herbal medicines were Korea Food and Drug Administration-certified and purchased from a local herbal market in South Korea; their botanical authenticity was confirmed by Prof. Dong Il Kim. A voucher specimen has been deposited at the Herbarium of the College of Korean Medicine, Dong-guk University, Ilsan, Korea. LASAP-C was extracted with $1 \mathrm{~L}$ distilled water at $100{ }^{\circ} \mathrm{C}$ for $4 \mathrm{~h}$ by using a Soxhlet extractor [12]. The extract was filtered through a filter paper (Hyundai Micro Co., Ltd., Korea), and the filtrate was freeze-dried (yield, $62 \mathrm{~g}$ ) and maintained at $4{ }^{\circ} \mathrm{C}$.

\section{Chemicals and reagents}

High-purity nitrogen gas was purchased from Shinyang Oxygen Co. (Seoul, South Korea). High-performance liquid chromatography-grade acetonitrile and acetic acid were purchased from Duksan Pure Chemicals Co. (Ansan, South Korea). 1-phenyl-2-thiourea (PTU) was purchased from Sigma (ST Louis, MO, USA) for the zebrafish study. Dulbecco's modified Eagle's medium (DMEM; SH30243.01), fetal bovine serum (FBS; SH30396.03), and penicillin-streptomysin solution (SV30010) were purchased from Hyclone Laboratories Inc. (Logan, UT, USA). Dimethyl sulfoxide (DMSO; D2650), kojic acid (K-3125), L-dopa (37830), and synthetic melanin (M-8631) were purchased from Sigma (St. Louis, MO, USA). All chemicals and reagents were of analytical grade.

The protease inhibitor cocktail Complete ${ }^{\mathrm{Tw}}$ was purchased from Roche (Mannheim, Germany). Protein assay reagent (\#500-0006) was purchased from Bio-Rad (Richmond, CA, USA). Tyrosinase (M-19, sc-7834), TRP-1 (M-19, sc-10448), TRP-2 (D-18, sc-10451), $\beta$ actin (I-19, sc-1616) antibodies, and commercially available secondary antibody were purchased from Santa Cruz Biotechnology, Inc. (Dallas, TX, USA).

\section{Ultra performance liquid chromatography tandem mass} spectrometry analysis

Before being injected to ultra performance liquid chromatography (UPLC) system, the LASAP-C extract was dissolved in distilled water to yield a $20 \mathrm{mg} / \mathrm{mL}$ concentration and filtered through $0.2 \mu \mathrm{m}$ syringe filter (Millipore, Bedford, MA, USA). UPLC-diode array detection analysis was performed using Waters ACQUITY Ultra Performance LC system equipped with Sample Manager-FTN, quaternary solvent manager, and photodiode array detector (Waters, Milford, MA, USA). The analysis was performed using ACQUITY UPLC HSS C18 column $(50 \times 2.1 \mathrm{~mm}$ i.d.; $1.8 \mu \mathrm{m})$. The UV detector monitoring wavelength was set to $300 \mathrm{~nm}$. The mobile phase consisted of acetonitrile and water acidified with $0.1 \%$ acetic acid (solvents A and B, respectively). The gradient program was as follows: 0 min, $1 \%$ of solvent $\mathrm{A} ; 2 \mathrm{~min}$, $1 \%$ of solvent A; 6 min, $20 \%$ of solvent A; 8 min, $20 \%$ of solvent A; $20 \mathrm{~min}, 35 \%$ of solvent A; $22 \mathrm{~min}, 100 \%$ of solvent A; $23 \mathrm{~min}, 1 \%$ of solvent A; $25 \mathrm{~min}, 1 \%$ of solvent $\mathrm{A}$, at a flow rate of $0.7 \mathrm{~mL}$ per minute. The injection volume was $0.5 \mu \mathrm{L}$.

AccuTOF ${ }^{\circ}$ single-reflectron time-of-flight mass spectrometer equipped with an electrospray ionization (ESI) source (JEOL, Peabody, MA, USA) was used for UPLCmass spectrometry (MS) study and was operated using Mass Center system version 1.3.7b (JEOL, Peabody, MA, USA). In the positive ion mode, the values were as follows: orifice $1=80 \mathrm{~V}$, ring lens $=10 \mathrm{~V}$, and orifice $2=5 \mathrm{~V}$. The ion guide potential and detector voltage were set to $2000 \mathrm{~V}$ and $2300 \mathrm{~V}$, respectively. ESI parameters were set as follows: needle electrode $=2000 \mathrm{~V}$, nitrogen gas flow rate as nebulizer $=1 \mathrm{~L} / \mathrm{min}$, nitrogen gas flow rate as desolvating agent $=3 \mathrm{~L} / \mathrm{min}$, desolvating chamber temperature $=250{ }^{\circ} \mathrm{C}$, and orifice 1 temperature $=80{ }^{\circ} \mathrm{C}$. Mass scale calibration was performed using YOKUDELNA calibration kit (JEOL, Tokyo, Japan) for accurate mass measurements and elemental composition calculations. MS acquisition range was set from $m / z 150$ to 2500 .

\section{B16F10 melanoma cell culture}

B16F10 melanoma cells derived from C57BL/6 J mouse were purchased from ATCC (Manassas, VA, USA). Cells were incubated in DMEM supplemented with $10 \% \mathrm{FBS}$, $100 \mathrm{U} / \mathrm{mL}$ penicillin, and $100 \mathrm{mg} / \mathrm{mL}$ streptomycin at $37{ }^{\circ} \mathrm{C}$ under $5 \% \mathrm{CO}_{2}$.

\section{Cell viability assay}

The safety of LASAP-C was determined by checking cell viability after treatment with various concentrations $(0,10,50,100,200$, and $400 \mu \mathrm{g} / \mathrm{mL})$ of LASAP-C by using 3-[4, 5-dimethylthiazol-2-yl]-2, 5 diphenyl tetrazolium bromide (MTT) colorimetric assay. This assay measures the metabolic reduction of MTT to formazan 
(blue) by mitochondrial dehydrogenase, which is active only in living cells [13]. B16F10 melanoma cells were incubated in 24-well plates at a density of $10^{4}$ cells/well for $24 \mathrm{~h}$. On the second day, cells were exposed to various concentrations of LASAP-C water extract for $48 \mathrm{~h}$. The media was then removed; the cells were washed with phosphate-buffered saline (PBS, $\mathrm{pH} 7.4$ ) and grown in $0.5 \mathrm{mg} / \mathrm{mL}$ MTT, which was prepared in PBS, at $37^{\circ} \mathrm{C}$. After $4 \mathrm{~h}$, the MTT reagent was removed, formazan crystals were dissolved in DMSO solution, and absorption values were read at $540 \mathrm{~nm}$ by using an enzyme-linked immunosorbent assay (ELISA) microplate reader (Bio-Tek Instruments Inc., Winooski, VT, USA).

\section{Measurement of cellular tyrosinase activity}

B16F10 melanoma cells $\left(5 \times 10^{4}\right.$ cells/well $)$ were seeded in a 6-well culture plate and treated with various concentrations $(10,50$, and $100 \mu \mathrm{g} / \mathrm{mL})$ of LASAP-C for $48 \mathrm{~h}$. They were washed with ice-cold PBS and lysed with phosphate buffer ( $\mathrm{pH}$ 6.8) containing $1 \%$ Triton X100. They were then disrupted by freezing and thawing, and whole cell lysates were clarified by centrifugation at $10,000 \mathrm{~g}$ for $5 \mathrm{~min}$. After protein levels were quantified and protein concentrations were adjusted using lysis buffer, $90 \mu \mathrm{L}$ of each lysate, containing the same amounts of protein, was placed in each well of a 96-well plate, and $10 \mu \mathrm{L}$ of $10 \mathrm{mML}$-DOPA was added. Control wells contained $90 \mu \mathrm{L}$ of lysis buffer and $10 \mu \mathrm{L}$ of $10 \mathrm{mM} \mathrm{L}$-DOPA. After incubation at $37^{\circ} \mathrm{C}$ for $15 \mathrm{~min}$, the dopachrome was observed by measuring the absorbance at $475 \mathrm{~nm}$ by using an ELISA reader.

Melanin content measurement in B16F10 melanoma cells The inhibitory effect of LASAP-C on the synthesis of melanin in B16F10 melanoma cells was evaluated by measuring melanin content in cells that were treated with three concentrations $(10,50$, and $100 \mu \mathrm{g} / \mathrm{mL})$ of LASAP-C for $48 \mathrm{~h}$. The cells were dissolved in $0.2 \mathrm{~mL}$ of $1 \mathrm{~N} \mathrm{NaOH}$ and boiled at $100{ }^{\circ} \mathrm{C}$ for $30 \mathrm{~min}$. The amount of melanin was determined by measuring absorbance at $460 \mathrm{~nm}$ by using the ELISA microplate reader and normalizing with protein level of cell lysates.

\section{Western blot analysis}

The effects of LASAP-C on the expression of tyrosinase, TRP-1, and TRP-2 were investigated using western blot analysis. The total amount of protein content of each supernatant was measured using Bradford assay (BioRad, Richmond, CA, USA). B16F10 melanoma cells were lysed in cell lysis buffer (62.5 mM Tris- $\mathrm{HCl}$ (pH 6.8), $2 \%$ sodium dodecyl sulfate (SDS), $5 \% \beta$-mercaptoethanol, protease inhibitor (Complete ${ }^{\mathrm{TM}}$, Roche, Mannheim, Germany), $2 \mathrm{mM}$ phenylmethylsulfonyl fluoride, $10 \mathrm{mM}$ EDTA, $50 \mathrm{mM} \mathrm{NaF}$, and $\left.1 \mathrm{mM} \mathrm{Na} \mathrm{VO}_{4}\right)$. Next, $10 \mu \mathrm{g}$ of protein per lane was separated by $8 \%$ SDS-polyacrylamide gel electrophoresis and blotted onto polyvinylidene fluoride membranes, which were then saturated with $5 \%$ non-fat dried milk in Tris-buffered saline containing $0.1 \%$ Tween 20 . Blots were exposed to specific primary antibodies at a dilution of 1:1000 overnight at $4{ }^{\circ} \mathrm{C}$, followed by incubation with a horseradish peroxidase-conjugated secondary antibody for $2 \mathrm{~h}$ at room temperature. The blots were developed using an enhanced chemiluminescence detection system (Thermo FisherScientific Inc., Rockford, IL, USA).

\section{Measurement of the effect of LASAP-C on pigmentation in zebrafish embryos}

The effect of LASAP-C on the pigmentation of zebrafish embryos was determined according to the method described previously [14]. Adult zebrafish (wild-type, AB) were obtained from a commercial dealer, and 10-15 fish were maintained in a $5 \mathrm{~L}$ acrylic tank under the following conditions: $28.5^{\circ} \mathrm{C}$, with a $14 / 10 \mathrm{~h}$ light/dark cycle. Zebrafish were fed three times a day and six days a week with Tetramin flake food (Tetra, Melle, Germany) supplemented with live brine shrimps (Artemia salina). Embryos were obtained from natural spawning, which was induced in the morning by turning on the light. The embryos were collected within $30 \mathrm{~min}$. Synchronized embryos were placed in 96-well plates containing $200 \mu \mathrm{L}$ embryo medium (13.7 mM NaCl, $5.4 \mathrm{mM} \mathrm{KCl,} 25 \mu \mathrm{M} \mathrm{Na}_{2} \mathrm{HPO}_{4}$,

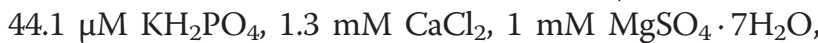
and $4.17 \mathrm{mM} \mathrm{NaHCO}_{3}$, ca. 10 drops $1 \mathrm{M} \mathrm{NaOH}$ to adjust $\mathrm{pH}$ 7.2). LASAP-C (10 and $100 \mu \mathrm{g} / \mathrm{mL}$ in $0.1 \%$ DMSO) was added to the embryo medium from $9 \mathrm{~h}$ post fertilization (hpf) to $72 \mathrm{hpf}$ (63 h exposure). Three embryos were assigned for each well. Further, $0.2 \mathrm{mM} 1$ phenyl-2-thiourea (PTU) was used to generate transparent zebrafish without interfering the developmental process [15]; these zebrafish were considered as a standard positive control. The effect on the pigmentation of zebrafish was observed under a stereomicroscope MZ16 (Leica Microsystems, Ernst-Leitz-Strasse, Germany). For observation, embryos were dechorionated using forceps, anesthetized in tricaine methane sulfonate solution (Sigma, St Louis, MO, USA), mounted in $3 \%$ methyl cellulose on a depression slide (Aquatic EcoSystem, Apopka, FL, USA), and photographed using stereomicroscope MZ16.

\section{Statistical analysis}

Results are presented as means \pm standard error of the mean of at least three independent experiments. Statistical analysis was performed using $t$-test. Student's $t$-test and paired $t$-test were performed to assess the differences between the means. $P$ values less than 0.05 were considered statistically significant. 


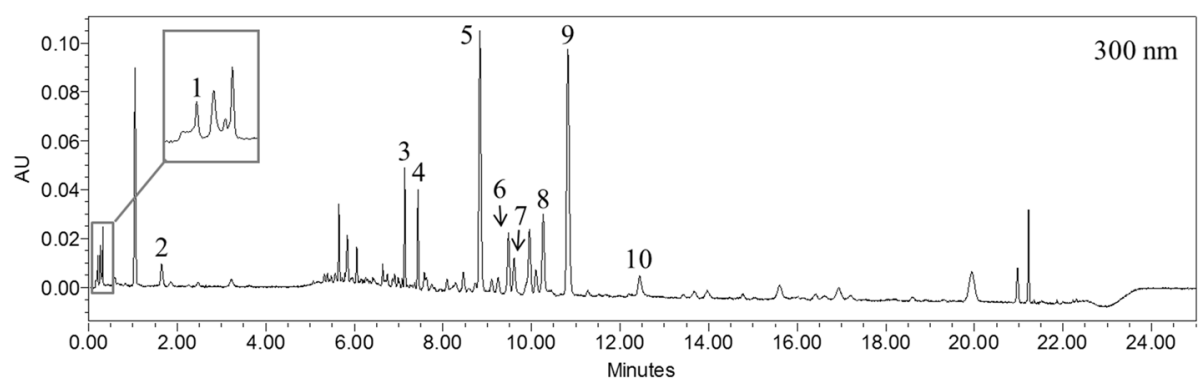

Fig. 1 Ultra-performance liquid chromatogram of LASAP-C

\section{Results and discussion}

\section{Establishment of the UPLC profile of LASAP-C extract}

The standard chromatogram for LASAP-C was established by using the UPLC system. The representative chromatogram of LASAP-C is shown in Fig. 1. Since several peaks were present as major components, other smaller peaks appeared close to the baseline. The major peaks of the chromatogram were identified by performing UPLC-ESI-MS.

\section{Identification of phytochemicals by UPLC-ESI-MS}

The retention time, observed mass, mass difference with theoretical mass, and proposed compounds of ten peaks are listed in Table 1. The ten phytochemicals in LASAP-C were as follows: betaine from Lycii Fructus; catalpol from Rehmanniae Radix Crudus; aviprin and byakangelicin from Angelicae dahuricae Radix; chrysin-6-Carabinose-8- $C$-glucose, chrysin-6- $C$-glucose- $8-C$-arabinose, baicalin, wogonin-7-O-glucuronide, oroxylin A-7-O-glucuronide, and baicalenin from Scutellariae Radix. These ten compounds were identified based on the UV and MS spectra of previously reproted. Although Rehmanniae Radix Crudus was the major component of LASAP-C in terms of quantity, phytochemicals from Scutellariae Radix were prominent as major peaks in the UPLC-MS study. The raw material of Rehmanniae Radix Crudus contained upto $77 \%$ of water contents depends on cultivars [16] and many primary metabolites such as saccharides, vitamins, and amino acids $[17,18]$. The most common iridoidal glycoside of raw Rehmanniae Radix Crudus is catalpol and its content in the dried root has been reported as 0.142 to $0.225 \%$ [16]. The high moisture content and a low catalpol content may lead to the representation as a minor peak in the UPLC-MS study.

Acteoside extracted from Rehmanniae Radix Crudus was reported to inhibit melanogenesis in B16F10 cells by the extracellular signal-regulated kinase (ERK) pathway [19] even though it was not detected in LASA-C total extract but valuable evidence of the skin whitening expectation on this herbal source. Aviprin and byakangelicin from Angelicae dahuricae Radix was identified as major peaks and byakangelicin was reported to inhibit melanogenesis without any influence on cell proliferation but aviprin has been reported to increase melanin production, in contrast [20]. However, Angelicae dahuricae Radix has been reported as a valuable skin whitening agent evidenced by the suppression of tyrosinase synthesis and inhibition of melanogenesis [20, 21]. It may be attributable to its various kinds of coumarin compounds which were

Table 1 The observed and calculated mass numbers of UPLC peaks of LASAP-C

\begin{tabular}{|c|c|c|c|c|c|c|c|}
\hline Peak No. & $\mathrm{RT}$ (min) & $\begin{array}{l}\text { Theoretical mass } \\
{[\mathrm{M}+\mathrm{H}]^{+}}\end{array}$ & $\begin{array}{l}\text { Observed mass } \\
{[\mathrm{M}+\mathrm{H}]^{+}}\end{array}$ & Mass difference (mmu) & Fragment ions & Identification & Reference \\
\hline 1 & 0.21 & 118.08680 & 118.08978 & 2.98 & - & betaine & [31] \\
\hline 2 & 1.65 & 362.12129 & 362.12673 & 5.44 & - & catalpol & [18] \\
\hline 3 & 7.14 & 549.16081 & 549.15920 & -8.96 & $531.15137 / 434.18988$ & chrysin-6-C-arabinose-8-C-glucose & [32] \\
\hline 4 & 7.44 & 549.16081 & 549.15912 & -9.04 & $531.15132 / 434.18978$ & chrysin-6-C-glucose-8-C-arabinose & [32] \\
\hline 5 & 8.84 & 447.09272 & 447.08914 & -3.58 & 271.06377 & baicalin & [32] \\
\hline 6 & 9.48 & 305.10195 & 305.10251 & 0.56 & 203.03895 & aviprin & [33] \\
\hline 7 & 9.56 & 335.11251 & 335.11512 & 2.61 & 233.05536 & byakangelicin & [33] \\
\hline 8 & 10.26 & 461.10782 & 461.10570 & -2.12 & 285.07676 & wogonin-7-O-glucuronide & {$[32]$} \\
\hline 9 & 10.82 & 461.10782 & 461.10585 & -1.97 & 285.07221 & oroxylin A-7-O-glucuronide & [32] \\
\hline 10 & 12.45 & 271.06065 & 271.06377 & 3.12 & - & baicalenin & [32] \\
\hline
\end{tabular}




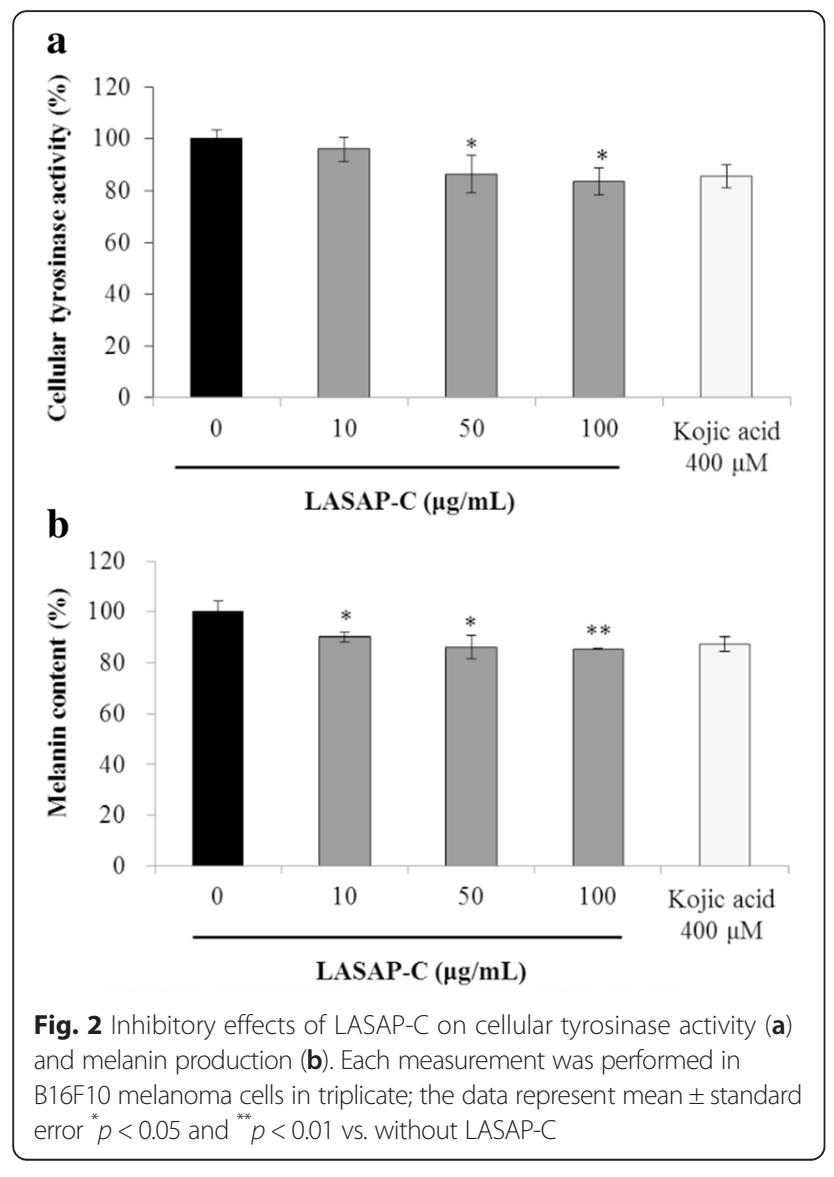

reported to have different effects on skin whitening. For example, aviprin (oxygenated linear-prenylfurocoumarin) exhibited weak stimulation of melanin production but phellopterin (linear-prenylfurocoumarin), heraclenin (oxygenated linear-prenylfurocoumarin), and columbianadin (acylated angular-dihydrofurocoumarin) suppressed melanogenesis [20]. Baicalin from Scutellariae Radix has been known to restrain melanogenesis of human melanocytes induced by ultraviolet [22].
Effects of LASAP-C on tyrosinase activity and melanin synthesis in B16F10 cells

The cytotoxicity of LASAP-C was determined by treating B16F10 cells with LASAP-C (0, 10, 50, 100, 200, and $400 \mu \mathrm{g} / \mathrm{mL}$ ) for 2 days and measuring the cell viability (data not shown). The LASAP-C extract did not induce any cytotoxicity up to $100 \mu \mathrm{g} / \mathrm{mL}$ concentration, but dose-dependently reduced viability at higher doses (53\% at $200 \mu \mathrm{g} / \mathrm{mL}, 68 \%$ at $300 \mu \mathrm{g} / \mathrm{mL}$, and $70 \%$ at $400 \mu \mathrm{g} / \mathrm{mL})$. According to the cell viability results, cells were treated with LASAP-C at a maximum concentration of $100 \mu \mathrm{g} / \mathrm{mL}$.

Tyrosinase is a rate-limiting enzyme in melanin synthesis. The biosynthesis of melanin is initiated by the catalytic oxidation of tyrosine to dopa by tyrosinase in the presence of dopa as a cofactor [23]. Tyrosinase activity of cell lysates treated with various concentrations (10, 50, and $100 \mu \mathrm{g} / \mathrm{mL})$ of LASAP-C was measured; dose-dependent reduction in tyrosinase activity was observed compared to that of the blank and positive control (400 $\mu \mathrm{M}$ kojic acid). The B16F10 cells retained 96.0, 86.02, and $83.7 \%$ tyrosinase activity after treatment with 10, 50, and $100 \mu \mathrm{g} / \mathrm{mL}$ LASAP-Cs (Fig. 2a). LASAP-C reduced cellular melanin content in B16F10 melanoma cells compared to that in the positive control (Fig. 2b). Cells cultured with 10, 50, and $100 \mu \mathrm{g} / \mathrm{mL}$ of LASAP-C showed significantly decreased levels of melanin (Fig. 2b). B16F10 cells retained 90.18, 86.02, and $85.53 \%$ melanin content after treatment with 10,50 , and $100 \mu \mathrm{g} / \mathrm{mL}$ LASAP-C.

Effects on the expression of tyrosinase, TRP-1, and TRP-2 (dopachrome tautomerase) proteins

Melanin is synthesized within melanosomes that contain three major pigment enzymes: tyrosinase, TRP-1, and TRP-2 [24-26]. The protein levels of tyrosinase, TRP-1, and TRP-2 in B16F10 cells were dose-dependently downregulated by treatment with LASAP-C extract (Fig. 3). The anti-melanogenesis activity of LASAP-C was shown
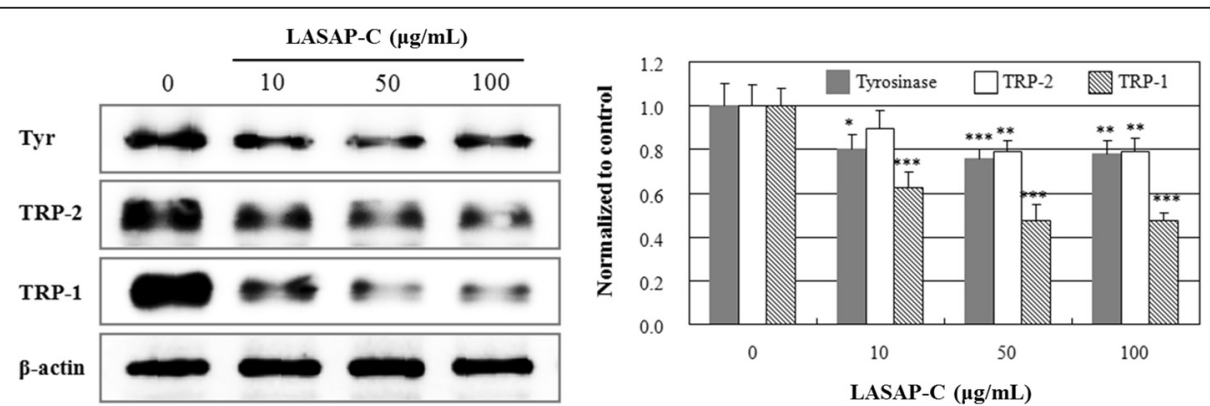

Fig. 3 The effects of LASAP-C extract on protein levels of melanin synthesis enzymes. The activities of tyrosinase, tyrosinase-related protein (TRP)-1, and TRP-2 in B16F10 melanoma cells were measured. Each determination was made in triplicate; the data represent mean \pm standard error. ${ }^{*} p<0.05$, ${ }^{* *} p<0.01$, and ${ }^{* * *} p<0.001$ vs. without LASAP-C 


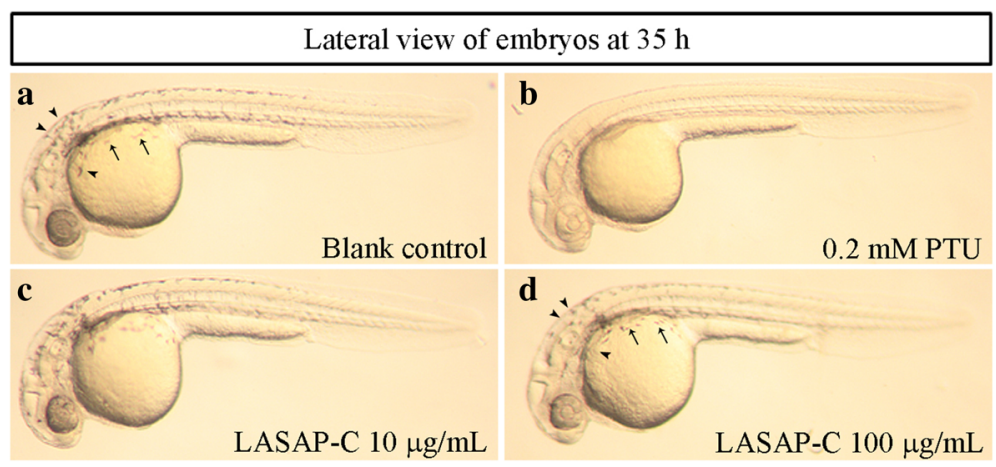

Fig. 4 The effects of LASAP-C extract on melanogenesis in zebrafish. Synchronized embryos were treated with 10 and $100 \mu \mathrm{g} / \mathrm{mL}$ LASAP-C. The effects on zebrafish pigmentation were observed using a stereomicroscope. In blank control and $100 \mu \mathrm{g} / \mathrm{mL}$ LACAP-C treated zebrafish, the pigmentation was pointed as black arrowhead and shrinkage of pigment cells was marked as black arrow. Lateral view of embryos at $31 \mathrm{~h}$ post fertilization: a blank control, b $0.2 \mathrm{mM}$ 1-phenyl-2-thiourea (PTU), c $10 \mu \mathrm{g} / \mathrm{mL}$ LASAP-C, and d $100 \mu \mathrm{g} / \mathrm{mL}$

to be directly related with the down-regulation of the major genes associated with the key enzymes involved in melanogenesis.

\section{Effects of LASAP-C on melanin synthesis in zebrafish}

Recently, the zebrafish model is being actively used in various in vivo assays as a good alternative system to rodents [27-29]. The effects on the pigmentation of zebrafish were determined stereomicroscopically. PTU, a well-known tyrosinase inhibitor, was used as a positive control of pigment production inhibition in zebrafish [30]. LASAP-C extract dose-dependently inhibited melanin synthesis in zebrafish embryos (Fig. 4c and d). The potency of the antimelanogenic effect of LASAP-C $(100 \mu \mathrm{g} / \mathrm{mL})$ was mild compare to the potency of $0.2 \mathrm{mM}$ PTU, the positive control. LASAP-C treatment not only reduced pigmentation (black arrowhead) but also caused shrinkage of pigment cells (black arrow) in Fig. 4.

\section{Conclusion}

In this study, the anti-melanogenic effect of LASAP-C was confirmed, and five major components of this traditional prescription were identified by UPLC-ESI-MS for quality control for further research and development.

The anti-melanogenic effect of LASAP-C extract was thought to occur via the inhibition of three key enzymes involved in melanin synthesis. The dose-dependent inhibitory effect of LASAP-C on tyrosinase, TRP-1, and TRP-2 expression indicated that this herbal prescription was directly related with the inactivation of genes encoding these proteins.

The whitening effect of LASAP-C was also validated using zebrafish as a whole-animal model for phenotypebased screening of LASAP-C extract. As expected, LASAP$\mathrm{C}$ extract remarkably inhibited body pigmentation in zebrafish embryos, reconfirming its anti-melanogenic efficacy. Taken together, these results suggest that LASAP-C has a strong potential to be used as an active ingredient in various cosmeceutical products for skin whitening.

\section{Abbreviations}

DMSO, dimethyl sulfoxide; L-DOPA, L-3,4-dihydroxyphenylalanine; PTU, 1phenyl-2-thiourea; ROS, reactive oxygen species; TRP-1, tyrosinase-related protein-1; TRP-2, tyrosinase-related protein-2; UV, ultraviolet

\section{Acknowledgments}

We would like to thank Editage (www.editage.com) for editing and reviewing this manuscript for English language.

\section{Funding}

The funding needed for the design of the study and collection, analysis, and interpretation of data and in writing the manuscript was supported by a grant from the Korea Healthcare Technology R\&D Project, Ministry of Health \& Welfare, Republic of Korea (Grant No.: HN12C0062).

\section{Availability of data and materials}

The datasets supporting the conclusions of this article are included within the article.

\section{Authors' contributions}

YPJ, SYC, JHL, and DIK participated in the design of this study. The herbal prescription was formulated and provided by MSC and DIK. The zebrafish experiment was performed and analysed by JHL, MYK, and HJR. The other experiments were conducted and manuscript was drafted by CYB and MKK. SYC and YPJ supervised the work and reviewed the draft and final manuscript and helped in the interpretation of results. All authors read and approved the final manuscript for submission.

\section{Competing interests}

The authors declare that they have no competing interests.

\section{Consent for publication}

Not applicable.

\section{Ethics approval and consent to participate}

The animal experiments were conducted in accordance with the National Institutes of Health Guide for the Care and Use of Laboratory Animals (NIH Publications No. 80-23), revised in 1996, and were approved by the Chungnam National University Institutional Animal Care and Use Committee (Approval No.: CNU-00535). 


\section{Author details}

'Department of Life and Nanopharmaceutical Sciences, College of Pharmacy, Kyung Hee University, Hoegi-dong, Dongdaemun-gu, Seoul 130-701, South Korea. ${ }^{2}$ Division of Pharmacognosy, College of Pharmacy, Kyung Hee University, Hoegi-dong, Dongdaemun-gu, Seoul 130-701, South Korea. ${ }^{3}$ Department of Preventive Pharmacy and Toxicology, College of Pharmacy, Kyung Hee University, Hoegi-dong, Dongdaemun-gu, Seoul 130-701, South Korea. ${ }^{4}$ Department of Dermatology, School of Medicine, Chungnam National University, 266 Munhwa-ro, Jung-gu, Daejeon 301-747, South Korea. ${ }^{5}$ Department of Biological Science, College of Bioscience and Biotechnology, Chungnam National University, 88 Daekak-ro, Yuseong-gu, Daejeon 305-764, South Korea. ${ }^{6}$ Dongguk University Bundang Oriental Hospital, 268 Buljeong-ro, Bundang-gu, Seongnam-si, Gyeonggi-do, South Korea. ${ }^{7}$ Dongguk University International Hospital, 27 Donkguk-ro, Ilsandong-gu, Goyang-si, Gyeonggi-do, South Korea.

Received: 26 January 2016 Accepted: 28 June 2016 Published online: 16 July 2016

\section{References}

1. Briganti S, Camera E, Picardo M. Chemical and instrumental approaches to treat hyperpigmentation. Pigment Cell Res. 2003;16:101-10.

2. Wang $\mathrm{K}-\mathrm{H}$, Lin R-D, Hsu F-L, Huang $\mathrm{Y}-\mathrm{H}$, Chang $\mathrm{H}-\mathrm{C}$, Huang $\mathrm{C}-\mathrm{Y}$, Lee $\mathrm{M}-\mathrm{H}$. Cosmetic applications of selected traditional Chinese herbal medicines. J Ethnopharmacol. 2006;106:353-9.

3. Parvez S, Kang M, Chung H-S, Bae H. Naturally occurring tyrosinase inhibitors: Mechanism and applications in skin health, cosmetics and agriculture industries. Phytother Res. 2007;21:805-16.

4. Fuchs J. Potentials and limitations of the natural antioxidants RRR-alphatocopherol, $L$-ascorbic acid and $\beta$-carotene in cutaneous photoprotection. Free Radic Biol Med. 1998;25:848-73.

5. Thiele JJ, Podda M, Packer L. Tropospheric ozone: an emerging environmental stress to skin. Biol Chem. 1997;378:1299-306.

6. Tadokoro T, Kobayashi N, Zmudzka BZ, Ito S, Wakamatsu K, Yamaguchi Y, Korossy KS, Miller SA, Beer JZ, Hearing VJ. UV-induced DNA damage and melanin content in human skin differing in racial/ethnic origin. FASEB J. 2003:17:1177-9.

7. Agar N, Young AR. Melanogenesis: a photoprotective response to DNA damage? Mutat Res/Fund Mol Mech Mut. 2005;571:121-32.

8. Wood SR, Berwick M, Ley RD, Walter RB, Setlow RB, Timmins GS. UV causation of melanoma in Xiphophorus is dominated by melanin photosensitized oxidant production. Proc Natl Acad Sci U S A. 2006;103:4111-5.

9. Wang H-T, Choi B, Tang M-S. Melanocytes are deficient in repair of oxidative DNA damage and UV-induced photoproducts. Proc Natl Acad Sci. 2010;107: 12180-5.

10. Sturm RA, Teasdale RD, Box NF. Human pigmentation genes: identification structure and consequences of polymorphic variation. Gene. 2001;277:49-62.

11. Ho C. Donguibogam: Principles and Practice of Eastern Medicine. Seoul: Ministry of Health, Welfare and Family Affairs; 2010.

12. Jensen WB. The origin of the Soxhlet extractor. J Chem Educ. 2007:84:1913.

13. Hansen MB, Nielsen SE, Berg K. Re-examination and further development of a precise and rapid dye method for measuring cell growth/cell kill. J Immunol Methods. 1989:119:203-10.

14. Choi T, Kim J, Ko D, Kim C, Hwang J, Ahn S, Kim S, Kim C, Lee J, Yoon T. Zebrafish as a new model for phenotype-based screening of melanogenic regulatory compounds. Pigment Cell Res. 2007;20:120-7.

15. Karlsson J, von Hofsten J, Olsson P-E. Generating transparent zebrafish: a refined method to improve detection of gene expression during embryonic development. Mar Biotechnol. 2001:3:522-7.

16. Park C, Lim J, Ryu K. Content of catalpol in Rehmanniae Radix and its preparations. Bull KH Pharma Sci. 1987;15:93-8.

17. Zhang R-X, Li M-X, Jia Z-P. Rehmannia glutinosa: review of botany, chemistry and pharmacology. J Ethnopharmacol. 2008;117(2):199-214.

18. You B-R, Kim H-R, Kim H-J, Lee J-Y, Lee S-Y, Song M-R, et al. Catalpol content and antioxidant activities in various cultivars of Rehmannia glutinosa. J Korean Soc Food Sci Nutr. 2011;40(4):481-5.

19. Son YO, Lee SA, Kim SS, Jang YS, Chun JC, Lee JC. Acteoside inhibits melanogenesis in B16F10 cells through ERK activation and tyrosinase downregulation. J Pharm Pharmacol. 2011;63(10):1309-19.

20. Matsuda $H$, Hirata N, Kawaguchi Y, Yamazaki M, Naruto S, Shibano M et al. Melanogenesis stimulation in murine B16 melanoma cells by umberiferae plant extracts and their coumarin constituents. Biol Pharm Bull. 2005;28(7): 1229-33.

21. Cho Y, Kim J, Park S, Lee B, Pyo H, Park H. New cosmetic agents for skin whitening from Angelica dahurica. J Cosmet Sci. 2006:57(1):11-22.

22. Zhou M, Li L, Zhou G-P. Study of restraint effect of baicalin on ultravioletinduced melanogenesis of human melanocytes in vitro. J Clin Dermatol Nanjing. 2002;31(10):613-4

23. Korner A, Pawelek J. Mammalian tyrosinase catalyzes three reactions in the biosynthesis of melanin. Science. 1982;217:1163-5.

24. Yokoyama K, Suzuki H, Yasumoto K-I, Tomita Y, Shibahara S. Molecular cloning and functional analysis of a cDNA coding for human DOPAchrome tautomerase/tyrosinase-related protein-2. Biochim Biophy Acta (BBA)-Gene Structure and Expression. 1994;1217:317-21.

25. Prota G. Some new aspects of eumelanin chemistry. Prog Clin Biol Res. 1987:256:101-24.

26. Kobayashi T, Urabe K, Winder A, Jimenez-Cervantes C, Imokawa G, Brewington T, Solano F, Garcia-Borron J, Hearing $\vee$. Tyrosinase related protein 1 (TRP1) functions as a DHICA oxidase in melanin biosynthesis. EMBO J. 1994;13:5818.

27. Nazario LR, Antonioli R, Capiotti KM, Hallak JEC, Zuardi AW, Crippa JAS, Bonan CD, da Silva RS. Caffeine protects against memory loss induced by high and non-anxiolytic dose of cannabidiol in adult zebrafish (Danio rerio). Pharmacol Biochem Behav. 2015;135:210-6.

28. Burgoyne T, O'Connor MN, Seabra MC, Cutler DF, Futter CE. Regulation of melanosome number, shape and movement in the zebrafish retinal pigment epithelium by OA1 and PMEL. J Cell Sci. 2015;128:1400-7.

29. Tokarz J, Moller G, de Angelis MH, Adamski J. Zebrafish and steroids: what do we know and what do we need to know? Journal Steroid Biochem Mol Biol. 2013;137:165-73.

30. Peterson RT, Link BA, Dowling JE, Schreiber SL. Small molecule developmental screens reveal the logic and timing of vertebrate development. Proc Natl Acad Sci. 2000;97:12965-9.

31. Shin H-D, Suh J-H, Kim J-H, Lee H-Y, Eom H-Y, Kim U-Y, et al. Determination of betaine in Fructus Lycii using hydrophilic interaction liquid chromatography with evaporative light scattering detection. B Korean Chem Soc. 2012;33(2):553-8.

32. Luo J-L, Lu F-L, Liu Y-C, LO C-F. Identification of Scutellaria Baicalensis in Traditional Chinese Medicine Preparations by LC/MS/MS Fingerprinting Method. J Food Drug Anal. 2012;20(4):887-99.

33. Li B, Zhang X, Wang J, Zhang L, Gao B, Shi S, et al. Simultaneous characterisation of fifty coumarins from the roots of Angelica dahurica by Off-line Two-dimensional high-performance liquid chromatography coupled with electrospray ionisation tandem mass spectrometry. Phytochem Anal. 2014;25(3):229-40.

\section{Submit your next manuscript to BioMed Central and we will help you at every step:}

- We accept pre-submission inquiries

- Our selector tool helps you to find the most relevant journal

- We provide round the clock customer support

- Convenient online submission

- Thorough peer review

- Inclusion in PubMed and all major indexing services

- Maximum visibility for your research

Submit your manuscript at www.biomedcentral.com/submit 